\title{
Survival and Marginal Bone Loss of Dental Implants Supporting Cad-Cam Angled Channel Restorations: A Split-Mouth Retrospective Study
}

\author{
Eduardo Anitua ${ }^{1,2}$ Sofía Fernández-de-Retana ${ }^{2}$ Mohammad Hamdan Alkhraisat ${ }^{2}$ \\ ${ }^{1}$ Clínica Eduardo Anitua, Vitoria, Spain \\ 2BTI Biotechnology Institute, Vitoria, Spain

\begin{abstract}
Address for correspondence Eduardo Anitua, MD, DDS, PhD, Clínica Eduardo Anitua, C/Jacinto Quincoces 39, Vitoria, 01007, Spain (e-mail: eduardo@fundacioneduardoanitua.org).
\end{abstract}

Eur J Dent 2020;14:194-199

\begin{abstract}
Objective The aim of this study was to determine whether the screw emergence angulation correction by computer-aided design (CAD)-computer-aided manufacturing (CAM) can influence implant survival and marginal bone stability.

Materials and Methods This was a controlled split-mouth retrospective study of angled channel restorations. The dental implants supporting the prosthesis were divided into the following two groups: the first group (Group 1) included the implants that required screw channel angulation, while the second group (Group 2) included the implants that did not require this correction to screw the prosthesis to the implant. The main outcome variables were implant survival and marginal bone loss (MBL).

Results A total of 68 dental implants placed in 22 patients were included in the final

Keywords

- dental implant

- screw emergence correction

- CAD-CAM

- fixed dental prosthesis

- dental prosthesis angulation cohort. The mean follow-up time was $39.65 \pm 15.20$ months. None of the studied implants failed during the follow-up period and the mean MBL was $-0.29 \pm 0.51 \mathrm{~mm}$ at the end of the follow-up. No statistical differences in the MBL were observed between the two groups of the study $(-0.18 \pm 0.51$ and $-0.23 \pm 0.58 \mathrm{~mm}$, respectively).

Conclusion The angulation of the screw channel with CAD-CAM technology resulted in good clinical outcomes and did not affect MBL. Thus, the angulated screw channel might be considered an alternative to face undesired screw emergencies. Future prospective clinical studies should confirm these results.
\end{abstract}

\section{Introduction}

During the last few decades, implant-supported fixed prostheses are a routinely used strategy to restore dental aesthetics and function. In this context, it has been reported that fixed, implant-supported restorations offer a predictable treatment modality, which significantly improves the quality of life for patients.

Fixed prostheses can be retained to the dental implant by cementation or screw retention. Among both alternatives, screw-retained prostheses present several advantages that could tip the balance, such as retrievability, need for minimal interocclusal space, and easier maintenance., ${ }^{2,3}$ However, the aesthetic result of the prosthetic rehabilitation may be hampered by an undesired screw channel emergence, for example, in the labial or buccal sides of the prosthesis. ${ }^{4}$

The placement of prefabricated angled transepithelial abutments may solve this undesired screw channel emergence. ${ }^{5}$ Nevertheless, angled abutments only offer a limited range of angulation, which may not be the appropriate for all cases. Furthermore, it was previously suggested that the angulation of abutments may increase the stress to the implant and the adjacent bone. ${ }^{6}$
DOI https://doi.org/ 10.1055/s-0040-1709895 ISSN 1305-7456.
(C)2020 Dental Investigation Society
License terms

() (1) $\ominus \circledast$ 
In this context, computer-aided design (CAD)-computeraided manufacturing (CAM) is a consolidated and continuously evolving technology ${ }^{7,8}$ which could be the answer to face the problem of inappropriate screw channel emergence. The CAD-CAM technology is able to customize the screw channel angulation at any direction and with a wide range of magnitudes (but with upper limit), optimizing the prosthetic design. ${ }^{9}$ These advantages, among others, resulted in the wider use of the CAD-CAM technology in clinical practice. ${ }^{9}$ Nevertheless, the implementation of CAD-CAM technology in the clinics is faster than the available studies reporting on the clinical outcomes.

Recently, in a split-mouth retrospective study, our group reported that the angulation of the screw channel by CAD-CAM did not result in a higher frequency of technical complications. ${ }^{10}$ To complement these results and increase the available clinical evidence regarding the use of CAD-CAM technology in dentistry, the aim of this study was to determine the effect of screw channel angulation on implant survival and marginal bone loss (MBL) in the same study cohort.

\section{Materials and Methods}

\section{Study Design}

The manuscript was prepared according to the Strengthening the Reporting of Observational studies in Epidemiology (STROBE) guidelines, which were adapted to cohort studies. ${ }^{11}$ This study was performed following the Helsinki Declaration regarding investigations with human subjects. The entire research was conducted in a single center in Vitoria, Spain. An exemption from the institutional review board's (IRB's) approval of the study protocol was granted by the author's institution, as it was a retrospective study, and the evaluated medical device had already been approved for clinical use. The analyzed datasheet did not contain any patients' identifiable data (anonymized datasheet).

The starting study cohort was previously published. ${ }^{10}$ This was a controlled split-mouth retrospective study of angled channel restorations. The dental implants supporting the prosthesis were divided into the following two groups: the first group (Group 1) included the implants that required screw channel angulation, while the second group (Group 2) included the implants that did not require this correction to screw the prosthesis to the implant. The inclusion criteria were partial or complete prostheses placed between November 2014 and December 2015, age $\geq 18$ years old, had received fixed dental prostheses with an angulation of the screw channel up to $30^{\circ}$, had at least one nonangulated piece in the same prosthesis, prostheses partially or totally placed in anterior positions, and time lapse between the implant placement and the final angulated prosthesis connection $\leq 12$ months. As exclusion criteria, cantilevered prostheses were excluded.

\section{Variables}

The predictor variable was the angulation of the crew channel (Group 1 vs. Group 2). The primary outcome was the implant survival rate that was assessed by time-to-event analyses. The secondary outcome was MBL. Other clinical variables were recorded as clinically relevant to evaluate the effect of the predictor variable (implant location, insertion torque, implant length, patient' sex and age, prosthesis type, and follow-up time).

\section{Surgical Technique}

Following a reflection of a full-thickness flap, implant sites were marked by the initial drill $(1.5 \mathrm{~mm}$ drill $)$ working at 850 to $1000 \mathrm{rpm}$ under irrigation. Implant site preparation was continued with diameter drills. ${ }^{12,13}$ The implant surface was biofunctionalized with plasma-rich ingrowth factors (BTI Biotechnology Institute; Vitoria, Spain) before placement in the bone. ${ }^{14,15}$ The implants evaluated in this study had a moderately rough and acid-etched surface. ${ }^{16}$

CAD-CAM technology was used to correct the angulation of the screw channel adapted to each clinical case with an upper limit of $30^{\circ}$ in chrome-cobalt ( $\left.\mathrm{Cr}-\mathrm{Co}\right)$ metal frameworks. The virtual design of the $\mathrm{Cr}-\mathrm{Co}$ structure was done based on extra-oral scanning (Imetric) performed for the cast. For this step, the body scans for the transepithelial abutments were connected.

The superstructure design was performed in exocad software and the crew channel was angulated with PowerShape CAD software (AUTODESK). A computer-steered 5-axis milling machine produced the $\mathrm{Cr}-\mathrm{Co}$ structure. The same workflow was performed for the centered locations, but without the screw channel angulation correction. The prosthesis screw was the same for both groups (Ti Black screw, BTI Biotechnology Institute). The screw insertion torque, as recommended by the manufacturer, was given a manual multitorque wrench.

\section{Data Collection Methods}

Clinical and radiographic data were reviewed to assess implant survival (presence of the implant at the last visit) and other relevant clinical data (implant location, insertion torque, implant length, sex and age, type of prosthesis, and time of follow-up). MBL at the bone level was calculated from the radiographic record when the prosthesis was connected and in the last available radiograph both mesially and distally for each implant supporting the prosthesis. MBL was calculated as the subtraction of the bone level in the last radiographic record and at the moment of the angulated prosthesis connection. The mean of the mesial and distal MBL was also calculated. MBL was measured using the Sidexis software (Dentsply Sirona; York, US) and the length of the implant was used as calibrator.

\section{Statistical Analysis}

Qualitative variables were described by calculating the absolute and relative frequency, and quantitative variables were described by the mean and standard deviation. The normal distribution of the data was verified with the help of the Shapiro-Wilk test. Qualitative variables were analyzed with a $\chi^{2}$ test. The Mann-Whitney test was used to analyze statistical interaction between quantitative and qualitative variables. Linear regression was selected to test the effect 
between quantitative variables. The statistical significance was set at $p<0.05$. SPSS v15.0 for Windows statistical software package (SPSS Inc. Released 2006. SPSS for Windows, Version 15.0. Chicago, SPSS Inc.) was used for statistical analyses.

\section{Results}

After the application of the inclusion/exclusion criteria, the final cohort included in this study contained 22 patients with 68 dental implants. Of these 68 dental implants, 34 implants belonged to Group 1 and the other 34 belonged to Group 2. All included prostheses had at least one angled and one nonangled piece as shown in - Fig. 1. The main follow-up of dental implants was $45.5 \pm 15.02$ months and the mean follow-up of the prostheses was $39.65 \pm 15.20$ months. The localization distribution of the dental implants can be observed in - Fig. 2.

The distribution of the variables between groups 1 and 2 can be observed in - Table1. In this analysis, a higher percentage of short dental implants, located mostly in the posterior regions, were present in Group 1.

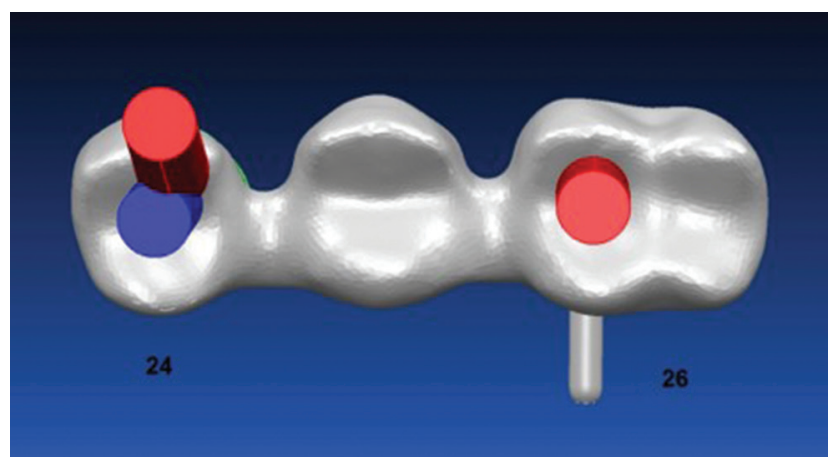

Fig. 1 Example of a prosthesis included in the study, observing angulation of the screw channel in position 24 (Group 1) and no need of angulation in position 26 (Group 2). The screw emergence is shown in red and screw emergence correction using CAD/CAM technology is shown in blue. CAD, computer-aided design; CAM, computer-aided manufacturing.

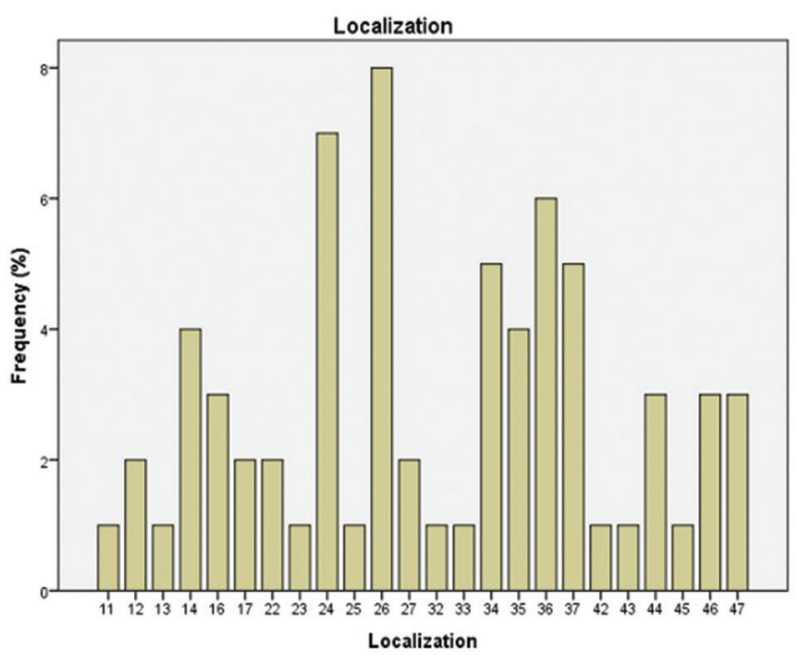

Fig. 2 Distribution of implant localization in the prostheses.
The dental implant survival at the end of the follow-up was $100 \%$. Regarding MBL, it was $-0.29 \pm 0.51 \mathrm{~mm}$ at the end of the follow-up. No statistical differences were observed between groups with regard to MBL.

Next, further confounding variables were evaluated in - Table 2. As observed, any of the recorded clinical variables were associated with MBL in the studied cohort.

\section{Discussion}

The aim of this study was to investigate the effect of correcting screw channel angulation by CAD-CAM on dental implant survival and MBL. The results observed in the studied cohort indicated that neither survival nor MBL were affected by the angulation of the screw channel. These results are in agreement with a previous publication with the same study cohort, where our group concluded that correcting the screw channel angulation by CAD-CAM does not result in an increased frequency of technical complications. ${ }^{10}$ To our knowledge, this was the first cohort comparing, with a split-mouth design, the clinical outcomes of angled prostheses by CAD-CAM.

Good clinical outcomes were observed for the angled prostheses, with a $100 \%$ dental implants' survival and low MBL. The MBL values observed in this study are within the ranges reported by other authors at similar follow-up times, ${ }^{17,18}$ supporting the use CAD-CAM technology to fabricate angled channel restorations.

In this context, the use of CAD-CAM technology could have several advantages in comparison with other available solutions to correct unfavorable implant positioning, such as cement-retained prostheses or placement of angled abutments. First, it was previously proposed in systematic reviews that screw-retained prostheses are associated with lower occurrence of biological complications, which are accompanied by higher retrievability. ${ }^{2,3}$ Second, regarding the use of angled abutments, the magnitude and direction of the screw channel angulation can be optimized to the clinical scenario. ${ }^{9}$ Third, it was previously proposed that restoring over angled abutments could result in negative consequences to the adjacent bone. In this sense, finite element analysis (FEA), comparing straight, and angled abutments reported higher strain in angled abutment independently of the bone type. ${ }^{6,19}$ Another study confirmed these results in another FEA, and they also observed that the stress was exacerbated when increasing the angulation of the prefabricated abutment from 0 to $20^{\circ} .^{20}$ These results were accompanied by in vitro studies, as the one recently published by Sousa et $a l,{ }^{21}$ who reported the removal torque of screws placed in straight abutments in comparison with angled abutments, together with the measurement of the von Misses stress quantification at the cervical level and the resistance to the single-load-to failure test. In this study, the authors observed lower removal torque of screws placed in angled abutments, together with higher von Misses stress at the cervical region and lower resistance to the single load-to failure test.

Finally, the results of these in vitro studies were also confirmed in the clinical field. A systematic review with 
Table 1 Description of the studied cohort with angulated or not angulated pieces

\begin{tabular}{|c|c|c|c|c|}
\hline Variable & Angulated piece & $\begin{array}{l}\text { Not angulated } \\
\text { piece }\end{array}$ & Total & Sign \\
\hline Dental implants (\%) & $34 / 68(50)$ & $34 / 68(50)$ & 68 & - \\
\hline Number of patients & $22 / 22$ & $22 / 22$ & 22 & - \\
\hline Age (mean $\pm S D$ ), years & - & - & $65.09 \pm 11.1$ & - \\
\hline \multicolumn{5}{|l|}{$\operatorname{Sex}(\%)$} \\
\hline Male & - & - & $12 / 22(54.54)$ & - \\
\hline Female & - & - & $10 / 22(45.45)$ & - \\
\hline $\begin{array}{l}\text { Follow-up time from implant insertion } \\
\text { (mean } \pm \text { SD), months }\end{array}$ & $44.32 \pm 16.37$ & $46.79 \pm 13.67$ & $45.5 \pm 15.02$ & $p=0.685$ \\
\hline $\begin{array}{l}\text { Follow-up time of the prosthesis (mean } \pm S D \text { ), } \\
\text { months }\end{array}$ & $38.32 \pm 16.5$ & $40.97 \pm 13.9$ & $39.65 \pm 15.20$ & $p=0.708$ \\
\hline \multicolumn{5}{|l|}{ Type of prosthesis } \\
\hline Partial (\%) & $32 / 34(94.1)$ & $30 / 34(88.2)$ & $32 / 68(91.17)$ & \multirow[t]{2}{*}{$p=0.393$} \\
\hline Complete (\%) & $2 / 34(5.9)$ & $4 / 34(11.8)$ & $6 / 68(8.83)$ & \\
\hline \multicolumn{5}{|l|}{ Implant length } \\
\hline Standard length (> $8 \mathrm{~mm}$ ) & $27 / 34(79.4)$ & 19/34 (55.9) & $46 / 68(67.64)$ & \multirow[t]{2}{*}{$p=0.038^{*}$} \\
\hline Short implants ( $\leq 8 \mathrm{~mm})$ & $7 / 34(20.6)$ & $15 / 37(44.1)$ & $22 / 68(32.35)$ & \\
\hline \multicolumn{5}{|l|}{ Localization } \\
\hline Maxilla (\%) & $16 / 34(47.1)$ & 19/34 (55.9) & $35 / 68(51.47)$ & \multirow[t]{2}{*}{$p=0.467$} \\
\hline Mandible (\%) & $18 / 34(52.9)$ & $15 / 34(44.1)$ & $33 / 68(48.53)$ & \\
\hline Anterior (\%) & $21 / 34(61.8)$ & $9 / 34(26.5)$ & $30 / 68(44.12)$ & \multirow[t]{2}{*}{$p=0.003^{* *}$} \\
\hline Posterior (\%) & $13 / 34(38.2)$ & $25 / 34(73.5)$ & $38 / 68(55.88)$ & \\
\hline Initial torque (mean $\pm \mathrm{SD}$ ), $\mathrm{Ncm}$ & $37.17 \pm 14.36$ & $37.32 \pm 18.1$ & $37.24 \pm 16.15$ & $p=0.684$ \\
\hline \multicolumn{5}{|l|}{$\mathrm{MBL}$} \\
\hline Mesial MBL (mean \pm SD), mm & $-0.12 \pm 0.66$ & $-0,18 \pm 0.61$ & $-0.14 \pm 0.63$ & $p=0.702$ \\
\hline Distal MBL (mean \pm SD), mm & $-0.25 \pm 0.59$ & $-0,29 \pm 0.73$ & $-0.27 \pm 0.66$ & $p=0.787$ \\
\hline Mean MBL (mean \pm SD), mm & $-0.18 \pm 0.51$ & $-0,23 \pm 0.58$ & $-0.29 \pm 0.51$ & $p=0.577$ \\
\hline Implant survival (\%) & 100 & 100 & 100 & $\mathrm{n} / \mathrm{a}$ \\
\hline
\end{tabular}

Abbreviation: MBL, marginal bone loss.

${ }^{*} p<0.05 ;{ }^{* *} p<0.01$.

Table 2 Statistical analysis of factors affecting MBL

\begin{tabular}{|c|c|c|c|}
\hline Variable & Mesial MBL & Distal MBL & Mean MBL \\
\hline Age (mean $\pm S D$ ), years & $\begin{array}{l}R=-0.241 \\
p=0.280\end{array}$ & $\begin{array}{l}R=-0.51 \\
p=0.82\end{array}$ & $\begin{array}{l}R=-0.137 \\
p=0.543\end{array}$ \\
\hline Sex (\%) & 0.46 & 0.36 & 0.408 \\
\hline Follow-up time from insertion (mean $\pm S D$ ), months & $\begin{array}{l}R=-0.084 \\
p=0.497\end{array}$ & $\begin{array}{l}R=-0.36 \\
p=0.836\end{array}$ & $\begin{array}{l}R=-0.035 \\
p=0.779\end{array}$ \\
\hline Follow-up time prosthesis (mean $\pm S D$ ), months & $\begin{array}{l}R=0.109 \\
p=0.376\end{array}$ & $\begin{array}{l}R=-0.16 \\
p=0.895\end{array}$ & $\begin{array}{l}R=0.056 \\
p=0.649\end{array}$ \\
\hline \multicolumn{4}{|l|}{ Types of prosthesis } \\
\hline Partial vs. complete & $p=0.204$ & $p=0.379$ & $p=0.705$ \\
\hline Implant length ( $\leq 8 \mathrm{~mm}$ vs. $>8 \mathrm{~mm}$ ) & 0.422 & 0.849 & 0.495 \\
\hline \multicolumn{4}{|l|}{ Localization } \\
\hline Maxilla/mandible & 0.234 & 0.649 & 0.436 \\
\hline Anterior/posterior & 0.775 & 0.762 & 0.951 \\
\hline Initial torque (mean $\pm \mathrm{SD}$ ), $\mathrm{Ncm}$ & $\begin{array}{l}R=-0.13 \\
p=0.329\end{array}$ & $\begin{array}{l}R=0.16 \\
p=0.902\end{array}$ & $\begin{array}{l}R=-0.72 \\
p=0.592\end{array}$ \\
\hline
\end{tabular}


meta-analysis compared the biological and mechanical complications associated with angled abutments by analyzing more than 4000 dental implants..$^{22}$ They observed that dental implants connected to angled abutments had higher probability of failure together with higher peri-implant MBL in comparison with straight abutments.

In addition, in order to interpret these results, it is important to consider that all the restorations analyzed in this cohort were indirect restorations, meaning that the prosthesis was screw-retained to prefabricated straight transepithelial abutments in contrast to direct to implant restorations, which was previously associated with lower MBL. ${ }^{23}$

Taking these results together, it can be proposed that the use of CAD-CAM technology is an alternative to correct the undesired screw channel emergencies, which result in good clinical outcomes. Keeping in mind the limitations, as this is a retrospective study, it is difficult to control the risk of bias in this study design. The split-mouth design followed in this study, in which each patient is its own control, could diminish the bias associated to retrospective studies. Nevertheless, these results should be confirmed with well-designed prospective clinical studies with longer time of follow-up and larger sample size.

\section{Conclusion}

The correction of screw channel angulation with CAD-CAM is not associated with an increased dental implant failure or MBL and it can be an alternative to other angled abutments or cemented restoration. After 3 years of follow-up, the angulation of the screw channel by CAD-CAM resulted in good clinical outcomes. Future prospective studies are required to confirm these results.

\section{Conflict of Interest}

E.A. is the Scientific Director of BTI Biotechnology Institute (Vitoria, Spain). He is the Head of the Foundation Eduardo Anitua, Vitoria, Spain. He also has the patent of PRGF technology. M.H.A. and S.F.d-R. are scientists at BTI Biotechnology Institute. M.H.A. reports personal fees from BTI Biotechnology Institute during the conduct of the study; personal fees from BTI Biotechnology Institute outside the submitted work. E.A. reports personal fees from BTI Biotechnology Institute during the conduct of the study; personal fees from BTI Biotechnology Institute outside the submitted work. In addition, he has two patents, plasma-rich ingrowth factors, and dental implant system, both licensed to BTI Biotechnology Institute. S.F.d-R. reports personal fees from BTI Biotechnology Institute during the conduct of the study; personal fees from BTI Biotechnology Institute outside the submitted work.

\section{References}

1 Nagahisa K, Arai K, Baba S. Study on oral health-related quality of life in patients after dental implant treatment with patient-reported outcome. Int J Oral Maxillofac Implants 2018;33(5):1141-1148
2 Wittneben JG, Millen C, Brägger U. Clinical performance of screw- versus cement-retained fixed implant-supported reconstructions-a systematic review. Int J Oral Maxillofac Implants 2014;29(Suppl):84-98

3 Sailer I, Mühlemann S, Zwahlen M, Hämmerle CHF, Schneider D. Cemented and screw-retained implant reconstructions: a systematic review of the survival and complication rates. Clin Oral Implants Res 2012;23(Suppl 6) :163-201

4 Gjelvold B, Sohrabi MM, Chrcanovic BR. Angled screw channel: An alternative to cemented single-implant restorations-three clinical examples. Int J Prosthodont 2016;29(1):74-76

5 Cavallaro J Jr, Greenstein G. Angled implant abutments: a practical application of available knowledge. J Am Dent Assoc 2011;142(2):150-158

6 Lin CL, Wang JC, Ramp LC, Liu PR. Biomechanical response of implant systems placed in the maxillary posterior region under various conditions of angulation, bone density, and loading. Int J Oral Maxillofac Implants 2008;23(1):57-64

7 Alikhasi M, Rohanian A, Ghodsi S, Kolde AM. Digital versus conventional techniques for pattern fabrication of implant-supported frameworks. Eur J Dent 2018;12(1):71-76

8 Bilgin MS, Baytaroğlu EN, Erdem A, Dilber E. A review of computer-aided design/computer-aided manufacture techniques for removable denture fabrication. Eur J Dent 2016;10(2): 286-291

9 Kapos T, Evans C. CAD/CAM technology for implant abutments, crowns, and superstructures. Int J Oral Maxillofac Implants 2014;29(Suppl):117-136

10 Anitua E, Flores C, Piñas L, Alkhraisat MH. Frequency of technical complications in fixed implant prosthesis: The effect of prosthesis screw emergence correction by computer-aided design/computer-aided manufacturing. J Oral Implantol 2018;44(6):427-431

11 von Elm E, Altman DG, Egger M, Pocock SJ, Gøtzsche PC, Vandenbroucke JP; STROBE Initiative. The Strengthening the Reporting of Observational Studies in Epidemiology (STROBE) statement: guidelines for reporting observational studies. J Clin Epidemiol 2008;61(4):344-349

12 Anitua E, Carda C, Andia I. A novel drilling procedure and subsequent bone autograft preparation: a technical note. Int J Oral Maxillofac Implants 2007;22(1):138-145

13 Anitua E, Alkhraisat MH, Piñas L, Orive G. Efficacy of biologically guided implant site preparation to obtain adequate primary implant stability. Ann Anat 2015;199:9-15

14 Anitua E, Tejero R, Zalduendo MM, Orive G. Plasma rich in growth factors promotes bone tissue regeneration by stimulating proliferation, migration, and autocrine secretion in primary human osteoblasts. J Periodontol 2013;84(8):1180-1190

15 Anitua E. Plasma rich in growth factors: preliminary results of use in the preparation of future sites for implants. Int J Oral Maxillofac Implants 1999;14(4):529-535

16 Anitua E, Orive G, Aguirre JJ, Ardanza B, Andía I. 5-year clinical experience with BTI dental implants: risk factors for implant failure. J Clin Periodontol 2008;35(8):724-732

17 Kim YT, Lim GH, Lee JH, Jeong SN. Marginal bone level changes in association with different vertical implant positions: a 3-year retrospective study. J Periodontal Implant Sci 2017;47(4):231-239

18 Segal P, Makhoul A, Eger M, Lucchina AG, Winocur E, Mijiritsky E. Preliminary study to evaluate marginal bone loss in cases of 2- and 3-implant-supported fixed partial prostheses of the posterior mandible. J Craniofac Surg 2019;30(4):1068-1072

19 Arun Kumar G, Mahesh B, George D. Three dimensional finite element analysis of stress distribution around implant with straight and angled abutments in different bone qualities. J Indian Prosthodont Soc 2013;13(4):466-472

20 Singh NK, Chalapathy SB, Thota RP, Chakravarthula K, Tirnati $\mathrm{R}$, Yenugupalli K. Evaluation of stress distribution among two 
different pre-angled abutments of implants in two different densities of bone at different levels along the implant-/n vitro study. J Contemp Dent Pract 2018;19(11):1370-1375

21 Sousa MP, Tribst JPM, de Oliveira Dal Piva AM, Borges ALS, de Oliveira S, da Cruz PC. Capacity to maintain placement torque at removal, single load-to-failure, and stress concentration of straight and angled abutments. Int J Periodontics Restorative Dent 2019;39(2):213-218
22 Omori Y, Lang NP, Botticelli D, Papageorgiou SN, Baba S. Biological and mechanical complications of angulated abutments connected to fixed dental prostheses. A systematic review with meta-analysis. J Oral Rehabil 2020;47(1):101-111

23 Hernández-Marcos G, Hernández-Herrera M, Anitua E. Marginal bone loss around short dental implants restored at implant level and with transmucosal abutment: a retrospective study. Int J Oral Maxillofac Implants 2018;33(6):1362-1367 